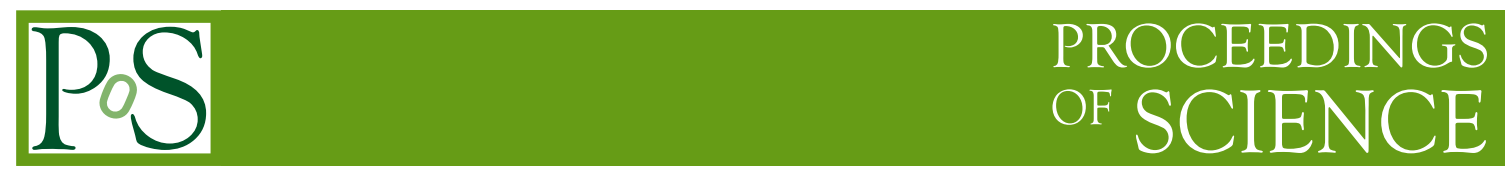

\title{
Study of hadron properties in cold nuclear matter with HADES
}

P. Tlusty ${ }^{* 14}$,G. Agakishiev5 , D. Belver ${ }^{15}$, A. Belyaev5 , J.C. Berger-Chen7, A. Blanco1, M. Böhmer ${ }^{8}$, P. Cabanelas ${ }^{15}$, A. Dybczak ${ }^{2}$, E. Epple ${ }^{7}$, L. Fabbietti ${ }^{7}$, P. Fonte ${ }^{1, a}$, J. Friese ${ }^{8}$, I. Fröhlich ${ }^{6}$, T. Galatyuk ${ }^{6, b}$, J. A. Garzón ${ }^{15}$, M. Golubeva ${ }^{10}$, D. González-Díaz ${ }^{c}$, F. Guber ${ }^{10}$, M. Gumberidze ${ }^{13}$, T. Hennino ${ }^{13}$, R. Holzmann ${ }^{3}$, P. Huck ${ }^{8}$, A. lerusalimov ${ }^{5}$, A. Ivashkin ${ }^{10}$, M. Jurkovic ${ }^{8}$, B. Kämpfer ${ }^{4, d}$, T. Karavicheva ${ }^{10}$, I. Koenig ${ }^{3}$, W. Koenig ${ }^{3}$, B. W. Kolb ${ }^{3}$, G. Korcyl ${ }^{2}$, G. Kornakov ${ }^{15}$, R. Kotte ${ }^{4}$, A. Krása ${ }^{14}$, F. Krizek ${ }^{14}$, H. Kuc ${ }^{2,13}$, A. Kugler ${ }^{14}$, A. Kurepin ${ }^{10}$, A. Kurilkin ${ }^{5}$, P. Kurilkin ${ }^{5}$, V. Ladygin ${ }^{5}$, R. Lalik ${ }^{7}$, S. Lang ${ }^{3}$, K. Lapidus ${ }^{7}$, T. Liu ${ }^{13}$, L. Lopes ${ }^{1}$, M. Lorenz ${ }^{6}$, L. Maier ${ }^{8}$, A. Mangiarotti ${ }^{1}$, J. Markert ${ }^{6}$, V. Metag $^{9}$, J. Michel ${ }^{6}$, C. Müntz ${ }^{6}$, R. Münzer ${ }^{7}$, L. Naumann ${ }^{4}$, M. Palka ${ }^{2}$, Y. Parpottas ${ }^{12,11}$, V. Pechenov ${ }^{3}$, O. Pechenova ${ }^{6}$, J. Pietraszko ${ }^{6}$, W. Przygoda ${ }^{2}$, B. Ramstein ${ }^{13}$, A. Reshetin ${ }^{10}$, A. Rustamov ${ }^{6}$, A. Sadovsky ${ }^{10}$, P. Salabura ${ }^{2}$, J. Siebenson ${ }^{7}$, Yu.G. Sobolev ${ }^{14}$, S. Spataro ${ }^{e}$, H. Ströbele ${ }^{6}$, J. Stroth ${ }^{6,3}$, C. Sturm ${ }^{3}$, A. Tarantola ${ }^{6}$, K. Teilab ${ }^{6}$, M. Traxler $^{3}$, H. Tsertos ${ }^{12}$, T. Vasiliev ${ }^{5}$, V. Wagner ${ }^{14}$, M. Weber $^{8}$, J. Wüstenfeld ${ }^{4}$, S. Yurevich ${ }^{3}$, Y. Zanevsky ${ }^{5}$ 


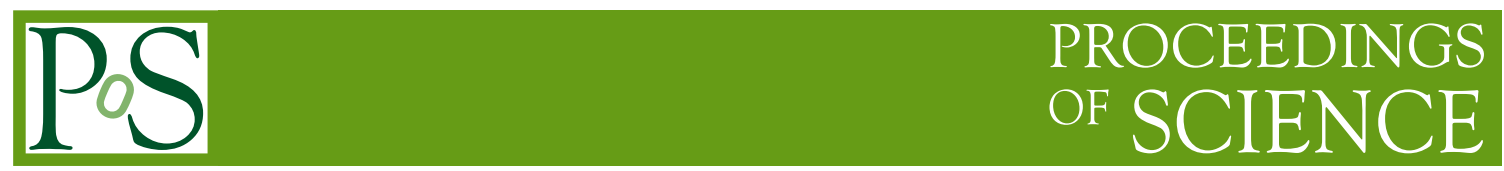

(HADES collaboration)

${ }^{1}$ LIP-Laboratório de Instrumentação e Física Experimental de Partículas , 3004-516 Coimbra, Portugal

${ }^{2}$ Smoluchowski Institute of Physics, Jagiellonian University of Cracow, 30-059 Kraków, Poland

${ }^{3}$ GSI Helmholtzzentrum für Schwerionenforschung GmbH, 64291 Darmstadt, Germany

${ }^{4}$ Institut für Strahlenphysik, Helmholtz-Zentrum Dresden-Rossendorf, 01314 Dresden, Germany

${ }^{5}$ Joint Institute of Nuclear Research, 141980 Dubna, Russia

${ }^{6}$ Institut für Kernphysik, Goethe-Universität, 60438 Frankfurt, Germany

${ }^{7}$ Excellence Cluster 'Origin and Structure of the Universe', 85748 Garching, Germany

${ }^{8}$ Physik Department E12, Technische Universität München, 85748 Garching, Germany

${ }^{9}$ II.Physikalisches Institut, Justus Liebig Universität Giessen, 35392 Giessen, Germany

${ }^{10}$ Institute for Nuclear Research, Russian Academy of Science, 117312 Moscow, Russia

${ }^{11}$ Frederick University, 1036 Nicosia, Cyprus

${ }^{12}$ Department of Physics, University of Cyprus, 1678 Nicosia, Cyprus

${ }^{13}$ Institut de Physique Nucléaire (UMR 8608), CNRS/IN2P3 - Université Paris Sud, F-91406 Orsay Cedex, France

${ }^{14}$ Nuclear Physics Institute, Academy of Sciences of Czech Republic, 25068 Rez, Czech Republic

${ }^{15}$ LabCAF. Dpto. Física de Partículas, Univ. de Santiago de Compostela, 15706 Santiago de Compostela, Spain

${ }^{a}$ also at ISEC Coimbra, Coimbra, Portugal

${ }^{b}$ also at ExtreMe Matter Institute EMMI, 64291 Darmstadt, Germany

${ }^{c}$ also at Technische Universität Darmstadt, Darmstadt, Germany

${ }^{d}$ also at Technische Universität Dresden, 01062 Dresden, Germany

${ }^{e}$ also at Dipartimento di Fisica Generale and INFN, Università di Torino, 10125 Torino, Italy

E-mail: tlusty@ujf.cas.cz

Results of a study of dielectron and pion production in $\mathrm{p}+\mathrm{Nb}$ collisions at incident proton beam energy $3.5 \mathrm{GeV}$ are presented. The data are taken by the High Acceptance Di-Electron Spectrometer (HADES) at GSI Darmstadt.

The dielectron invariant mass distributions are compared to data from elementary $p+p$ reactions at the same beam energy. At this energy above the light vector meson production threshold, the omega peak is clearly identified. The collected statistics and high acceptance for pair momenta within the interval 50-2000 MeV/c allow for a study of momentum dependence of the omega yield and spectral shape. The strongest medium modification in the line shape is expected for low momenta mesons decaying inside the nuclear matter. Comparing the measured $\mathrm{p}+\mathrm{Nb}$ data with the $\mathrm{p}+\mathrm{p}$ reference experiment results, the significant change in yield of low momentum dielectron pairs in the vector meson mass region is observed.

Data on the emission of charged pi mesons, which are related to neutral pions representing a dominant contribution to the dielectron yield, are shown as well. They contribute to the results from systematic studies of pion production in proton-nucleus collisions, which point to a transition of the pion source from simple NN collisions to the emission of thermalized pions from a fireball, when increasing the atomic number of the target nucleus. The data also serve as a reliable tool for normalization of the dielectron data.

50th International Winter Meeting on Nuclear Physics

23-27 January 2012

Bormio, Italy

${ }^{*}$ Speaker. 


\section{Introduction}

The dependence of hadron properties on medium effects represents one of the most important open problems of modern nuclear and particle physics. From a fundamental point of view, the partial restoration of the QCD chiral symmetry is expected to lead to a mass modification of vector mesons at finite temperature and/or finite nuclear density.

The QCD inspired models predict a change of the meson spectral function based on the chiral condensate modification by about $30 \%$ already at nuclear saturation density [1, 2, 3]. Similarly, a principially different approach of hadronic many-body models $[1,4]$ also gives prediction of significant modifications in spectral function shape of vector mesons, like $\rho, \omega$ and $\phi$, when embedded in nuclear matter.

Di-leptonic decay channels of vector mesons are well suited for the investigation of nuclear collisions because leptons do not interact strongly and carry out undistorted information about the properties of a meson decaying in the dense and hot nuclear matter. On the other hand, electromagnetic signals are very difficult to handle mainly due to the huge hadron background and low branching ratio for electromagnetic channels.

Di-lepton spectra measured at the CERN SPS in several experiments $[5,6,7]$ at various energies up to $158 \mathrm{AGeV}$ indicate significant in-medium modifications of the $\rho$ meson spectral shape. At lower energies (1-2 AGeV) the DLS experiment [8] and recently the HADES collaboration $[9,10,11]$ systematically measured dilepton production from elementary collisions, light and medium-size collision systems. In the medium size system of $\mathrm{Ar}+\mathrm{KCl}$ at $1.75 \mathrm{~A} \mathrm{GeV}$, an excess of the dielectron yield as compared to the elementary pp and np systems at intermediate masses below the $\rho / \omega$ mass is observed.

Another possibility to study hadron properties inside nuclear matter are proton, pion or photon induced reactions. In this case one explores a medium at ground state density and zero temperature where the predicted modifications of the meson spectral function is smaller than at higher densities. On the other hand, the advantage is that the system is well defined as it does not undergo a complicated density and temperature time evolution as in the nucleus-nucleus collision. The $\rho$ meson spectral shape from cold nuclear matter was measured by the CLAS experiment at JLab [12] and the KEK-E325 experiment at [13]. However, both experiments were not sensitive to low dilepton pair momenta where the predicted effects are strongest, and the results are not conclusive so far. For the $\omega$ and $\phi$ meson several experiments $[14,15,16,17]$ reported a sizable broadening of the total decay width inside the medium in the yield measurements using the transparency ratio method [18].

In this paper we present preliminary results on inclusive dielectron pair production in proton induced reactions on the $\mathrm{Nb}$ target at $E_{k i n}=3.5 \mathrm{GeV}$ measured with the HADES spectrometer [19]. The reference spectrum was obtained in $p+p$ reactions measured at the same energy [20]. The data taken with high statistics and acceptance covering the low pair momenta are sensitive to expected spectral shape changes as well as yield suppression due to collision broadening.

We also present data on the analysis of charged pion production. The results contribute to the data from systematic studies of pion production in proton-nucleus collisions (see e.g. [21]), and serve as a reliable tool for normalization of the dielectron data obtained in the same experiment.

The High Acceptance DiElectron Spectrometer HADES is devoted mainly to study the production 
of dielectron pairs from proton, pion and nucleus induced reactions at 1-2 AGeV. At the same time, the spectrometer provides detection and high quality identification of charged particles in a large solid angle.

\section{Experiment}

HADES [19] is a magnetic spectrometer designed as second-generation device for measurements of $e^{+} e^{-}$pairs. The spectrometer is segmented into six identical sectors that cover laboratory polar angles between 18 and 85 degrees. Its large azimuthal acceptance covers between $65 \%$ and $90 \%$ of $2 \pi$ at small and large polar angles, respectively. A fast hadron-blind Ring Imaging CHerenkov counter (RICH) is used for electron and positron identification. Four planes of Multi-wire Drift Chambers (MDC I - MDC IV), together with a superconducting magnet, form the magnetic spectrometer for track reconstruction and momentum determination. In the region behind the magnetic field, a set of electromagnetic Pre-Shower detectors (at polar angles $18^{\circ}-45^{\circ}$ ) and a time-of-flight wall are installed which form the META (Multiplicity and Electron Trigger Array). The time-of-flight detector wall is subdivided into 2 regions: TOF (at polar angles $45^{\circ}-85^{\circ}$ ), consisting of 384 scintillator slabs of varying length, which are read out at both ends, with a timeof-flight resolution of $\sigma=150 \mathrm{ps}$, and TOFINO (at polar angles $18^{\circ}-45^{\circ}$ ), consisting of 24 scintillator plates readout on one end, with a time-of-flight resolution of $\sigma=450 \mathrm{ps}$. The TOFINO is placed directly in front of the Pre-Shower detector, which provides precise position measurement. The TOF/TOFINO detectors are also used for fast charged-particle multiplicity measurements. Together with the Pre-Shower detectors they provide additional lepton/hadron discrimination power and track coordinate measurements with a spatial resolution in the range from 14 to $25 \mathrm{~mm}$.

In the reference pp experiment, a proton beam of $10^{7}$ particles/s with a kinetic energy of 3.5 $\mathrm{GeV}$ was incident on a $4.4 \mathrm{~cm}$ long liquid hydrogen target. For details on the experiment and results see [20].

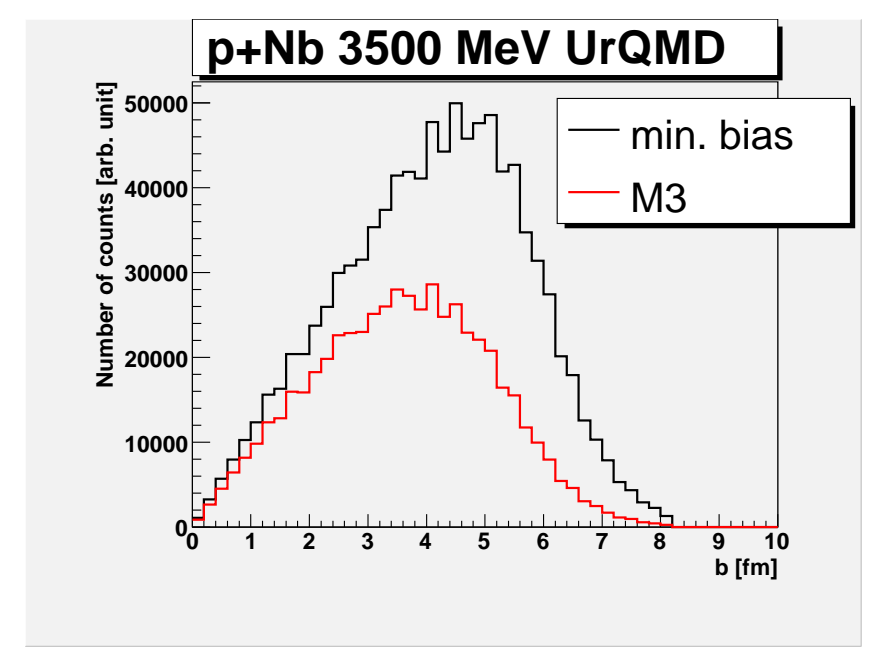

Figure 1: Impact parameter distribution for all (“minimum bias") and LVL1 accepted reactions 
In the $\mathrm{p}+\mathrm{Nb}$ experiment a beam of protons with kinetic energy $3.5 \mathrm{GeV}$ and intensity $2 \times 10^{7}$ per 10 second spill was incident on a segmented $\mathrm{Nb}$ target (12 rings, each $0.45 \mathrm{~mm}$ thick) with $2.9 \%$ total nuclear interaction length. The presented data were collected under the LVL1 trigger condition which was based on a fast determination of the charged-particle multiplicity $\left(M_{c h}\right)$ in the time-of-flight detectors. Events with $M_{c h} \geq 3$ were selected.

The LVL1 trigger bias was obtained from UrQMD transport model [22] calculations followed by full scale GEANT-3 [23] simulations of the detector setup. Fig. 1 shows the simulated impactparameter distributions. For the "minimum bias" events corresponding to the total reaction cross section we require in UrQMD at least one nuclear interaction. Then we pass these events through our analysis code and require that they fulfill the LVL1 condition. LVL1-triggered events correspond to $56 \%$ of the total reaction cross section, however one can see that selection of more "central" reaction is rather weak. Still this condition enhances the averaged multiplicity for e.g. $\pi^{-}$by $42 \%$ which has to be taken into account in the data analysis, see following sections.

Resulting dilepton cross sections were obtained via the comparison of the measured yield of a known physical process inside the HADES acceptance and its measured cross section. In $\mathrm{p}+\mathrm{p}$ collisions it was obtained via the exclusive measurement of elastic $\mathrm{p}+\mathrm{p}$ collisions and the known integrated cross section inside the HADES acceptance. In $\mathrm{p}+\mathrm{Nb}$ collisions such exclusive measurement was not possible. The physical source chosen there is the production of negative pions in proton induced reactions on nuclei. This was measured recently by the HARP-CDP collaboration [21] for a number of nuclei and projectile energies.

\section{Charged pions}

Due to the high intensity of the proton beam we did not use the diamond detector detecting individual beam particles, which usually provides the "start" time for time-of-flight measurement. Therefore the energy loss of particles detected in the time-of-flight TOF/TOFINO detectors was used for the particle identification.
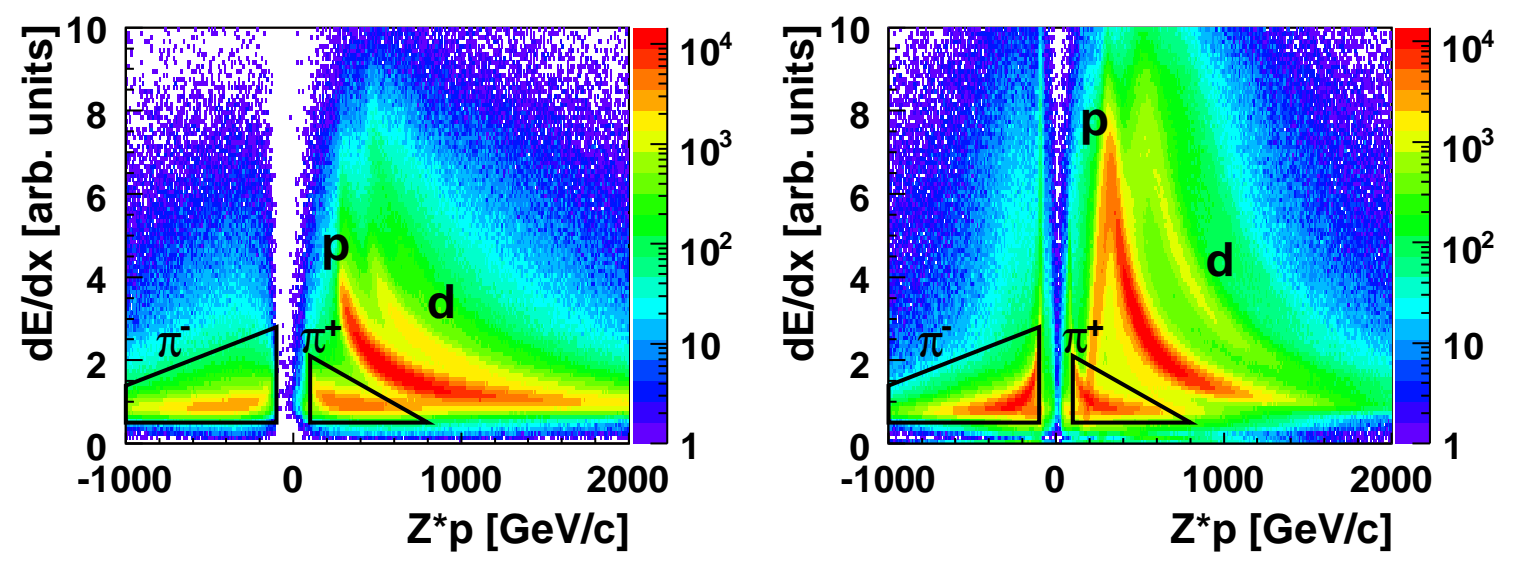

Figure 2: Distribution of the energy loss versus charge*momentum of particles detected in inner TOFINO (left) and outer TOF (right) detectors

Fig. 2 shows the distribution of the energy loss versus charge $\times$ momentum, together with cuts selecting regions with positive and negative pions. $\pi^{-}$mesons can be identified with high purity 
in a large interval of momenta $p \geq 150 \mathrm{MeV} / c$, while the range for $\pi^{+}$is limited to the region $150 \leq p \leq 600 \mathrm{MeV} / c$ due to an overlap with protons at higher momenta. Moreover, at momenta $\approx 200 \mathrm{MeV} / \mathrm{c}$ the energy loss of protons stopped in the scintillators of the outer TOF detector is the same as for $\pi^{+}$, which makes the identification in this region difficult. For this reason we limit ourself in this paper only on results on negative pions.

After the particle identification was done for all tracks, the resulting yields were corrected for efficiency and purity of the PID method, as well as for the detector and tracking efficiencies. The detection/tracking efficiency has been obtained from Monte Carlo simulations. Additionally an acceptance correction in azimuthal angle was applied.
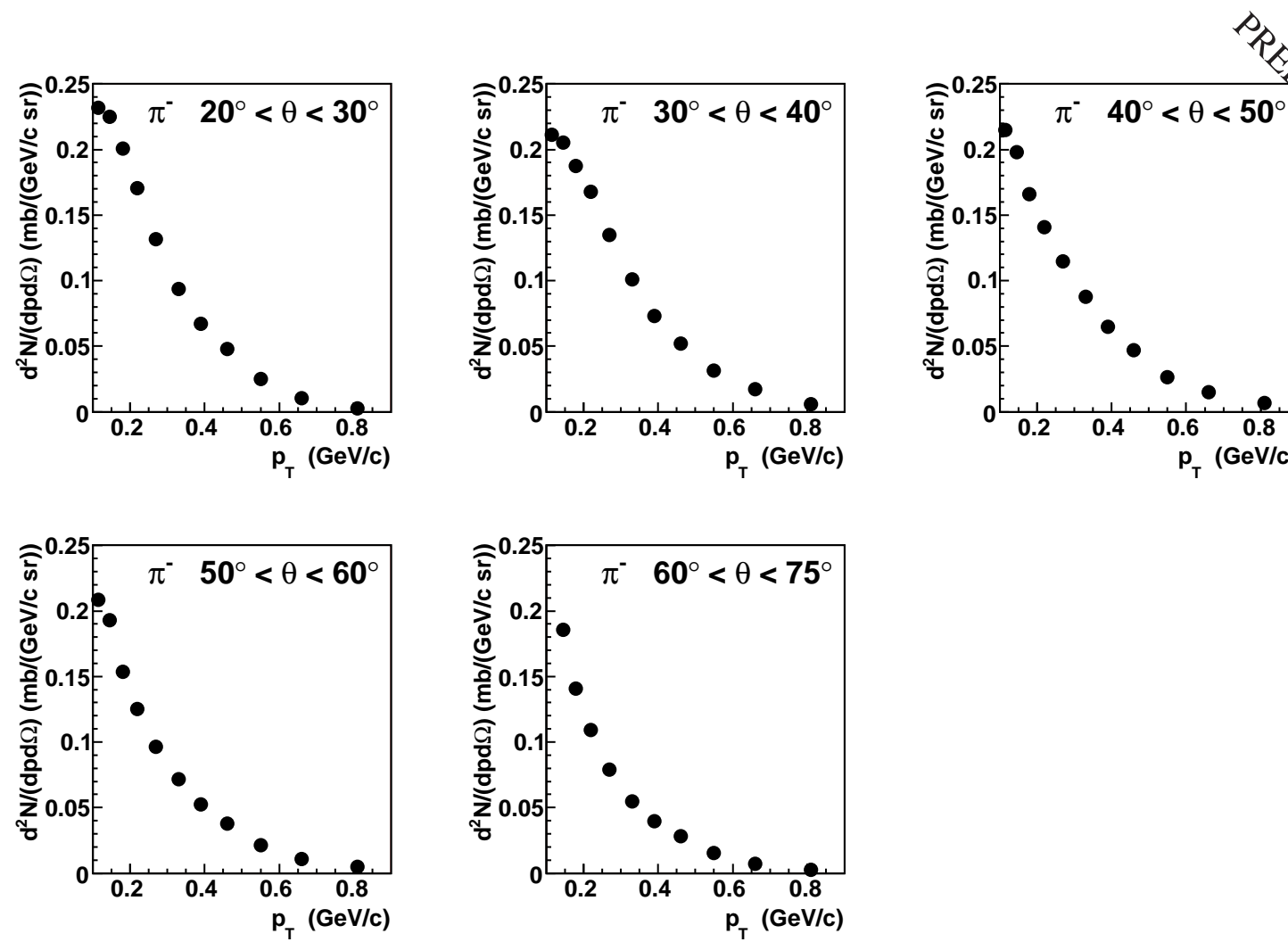

Figure 3: Multiplicity distribution of the $\pi^{-}$measured in various polar angle bins. Data are corrected for the LVL1 trigger bias. Only statistical errors are shown.

The multiplicity enhancement due to the LVL1 trigger bias of $42 \%$ was taken into account by the correction factor $1.42 \pm 0.14$. The systematic error of $10 \%$ was estimated from different scaling factors obtained from experiment and simulation, for two LVL1 trigger settings of $M_{c h} \geq 2$ and $M_{c h} \geq 3$.

The resulting pion multiplicities, corrected for the LVL1 trigger bias, are shown in Fig. 3. In order to obtain the normalization factor needed for determination of the dilepton pair production cross section, the resulting pion multiplicities are compared to existing data from a systematic study of pion production from $\mathrm{p}+\mathrm{A}[21]$. The system $\mathrm{p}+\mathrm{Nb}$ was not measured but the systematics allows for an interpolation for our collision system using two reactions $\mathrm{p}+\mathrm{Cu}$ and $\mathrm{p}+\mathrm{Ta}$ at closest measured energies 2.20 and $4.15 \mathrm{GeV}$. To interpolate the HARP-CDP data to our reaction system, 
a global scaling factors of $2.00,0.96,1.16$, and 0.48 were applied to the $2.20 \mathrm{GeV} \mathrm{p}+\mathrm{Cu}, 4.15 \mathrm{GeV}$ $\mathrm{p}+\mathrm{Cu}, 2.20 \mathrm{GeV} \mathrm{p}+\mathrm{Ta}$, and $4.15 \mathrm{GeV}$ p+Ta data sets, respectively.

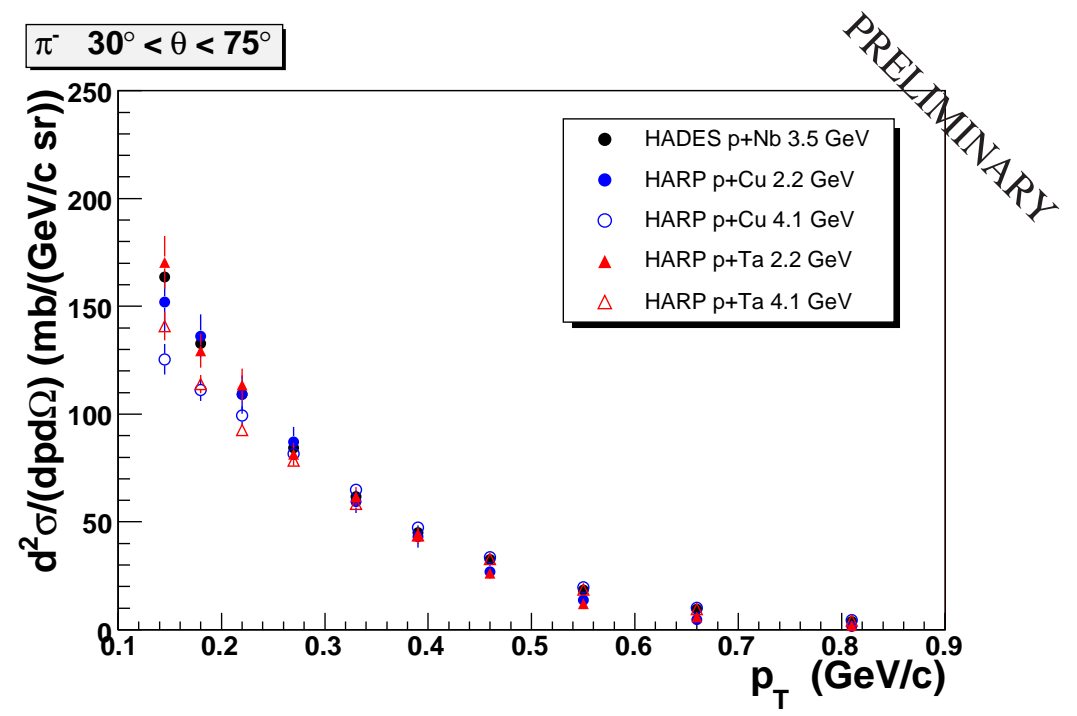

Figure 4: Inclusive cross section for $\pi^{-}$production in $\mathrm{p}+{ }^{93} \mathrm{Nb}$ collisions at $3.5 \mathrm{GeV}$ in the polar angle range $20^{\circ} \leq \theta \leq 75^{\circ}$.

Then we scaled the HADES multiplicity data to the HARP cross section. The scaling factor $\sigma_{R}=\sigma_{\pi^{-}}(H A R P) / M_{\pi^{-}}(H A D E S)$ is in fact the total reaction cross section for $\mathrm{p}+{ }^{93} \mathrm{Nb}$ at $3.5 \mathrm{GeV}$. The result of this is shown in Fig. 4. The scaling factor was obtained from the pion multiplicity by fitting the HADES data points multiplied by the scaling factor bin-by-bin to the HARP-CDP results.

As seen in Fig. 4, the cross section shapes for HARP data at higher energy differ at low momenta. The origin of this dicsrepancy needs to be investigated. Therefore, only a region of $p_{T}>300 \mathrm{MeV} / \mathrm{c}$ was used for the normalization. The resulting total reaction cross section is $848 \mathrm{mb}$. Statistical errors are negligible, while the systematic error is estimated as $15 \%$, with uncertainty of the trigger enhancement factor as a main source. The result differs by $14 \%$ from the value $982 \mathrm{mb}$ calculated by a parametrization of the total absorption cross section [24].

\section{Di-electrons}

All possible combinations of identifed e+e- tracks have been formed event by event and corrected for detector and reconstruction efficiencies. The latter ones were deduced using Monte-Carlo simulations embedded into real events. Invariant mass spectra of the unlike-sign pairs were constructed from single $e^{+} e^{-}$tracks. To increase the purity of the $e^{+} e^{-}$sample a cut on the single track momentum $0.08<p_{e} /(\mathrm{GeV} / \mathrm{c})<2.00$ was applied. The combinatorial background (CB) was extracted from all like-sign pair combinations inside the same event. Since the CB stems predominantly from external conversion it could be reduced by cutting on the pair opening angle > 9 degrees and on the track fitting quality. By subtracting the CB from the unlike-sign pairs the signal spectrum was obtained. The signal to background ratio is $S / B \approx 1$ at $M_{e e}=200 \mathrm{MeV} / \mathrm{c}^{2}$ and 
increases up to $S / B \approx 10$ at $M_{e e}=750 \mathrm{MeV} / \mathrm{c}^{2}$. The dielectron cross section was obtained from the measured multiplicities based on the $\pi^{-}$production analysis, see previous section. The data were also corrected for the $e^{+} e^{-}$multiplicity enhancement due to the LVL1 trigger, estimated from the simulations as 1.08 .
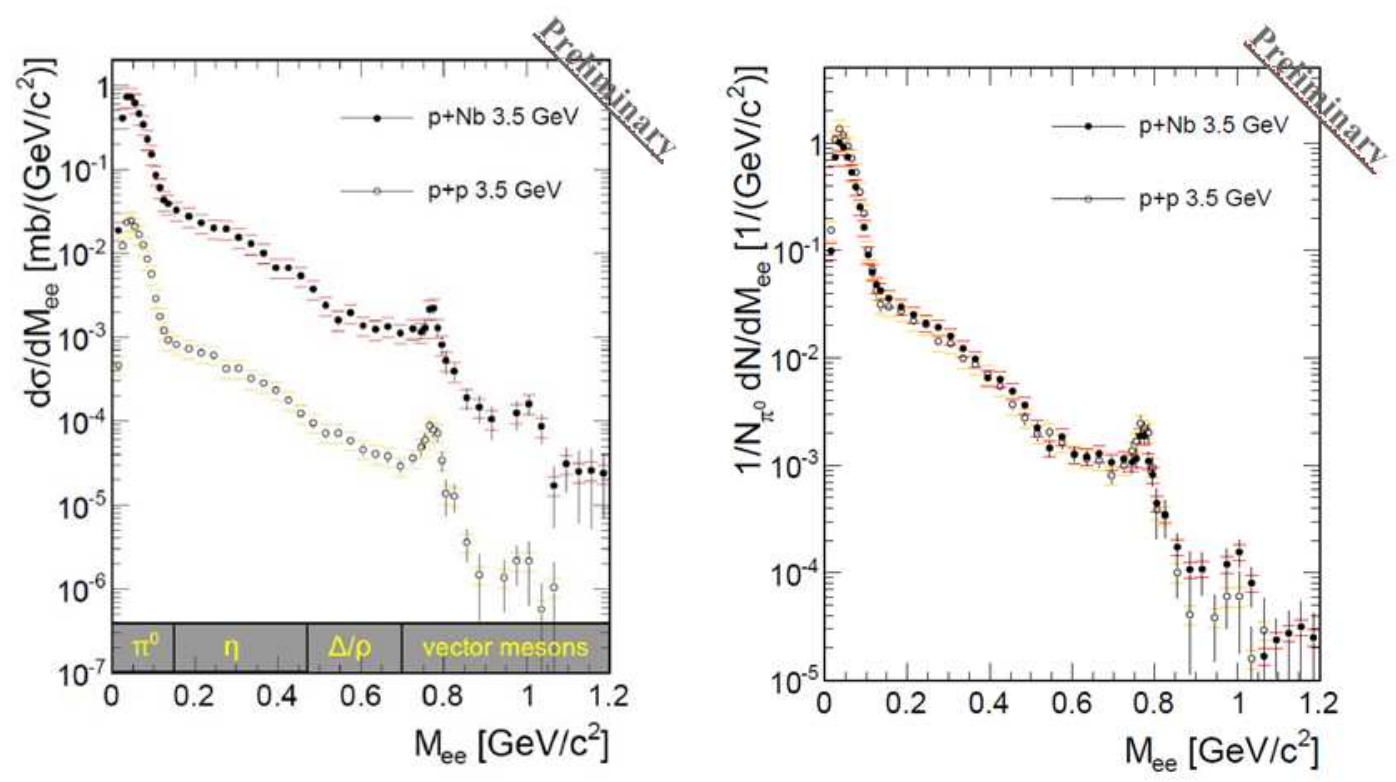

Figure 5: Inclusive $e^{+} e^{-}$invariant mass distributions from $\mathrm{p}+{ }^{93} \mathrm{Nb}$ and $\mathrm{p}+\mathrm{p}$ collisions at $3.5 \mathrm{GeV}$.

The measured invariant mass distributions of $e^{+} e^{-}$pairs from $\mathrm{p}+\mathrm{Nb}$ and $\mathrm{p}+\mathrm{p}$ reactions are shown in the left panel of Fig. 5. Systematic errors od $21 \%$ and $20 \%$ for $\mathrm{p}+\mathrm{Nb}$ and pp, resepectively, are displayed by the coloured horizontal bars. The low-mass part of the spectrum is dominated by $\pi^{0}$ Dalitz decays $\left(M_{e e}<150 \mathrm{MeV} / \mathrm{c}^{2}\right)$, while the intermediate part $\left(150 \mathrm{MeV} / \mathrm{c}^{2}<\right.$ $M_{e e}<550 \mathrm{MeV} / \mathrm{c}^{2}$ ) can be attributed to $\eta$ and $\Delta(1232)$ Dalitz decays. The high mass part of the distribution is dominated by the direct decay of the light vector mesons $\omega, \rho$ and $\phi$. The peak around $780 \mathrm{MeV} / \mathrm{c}^{2}$ corresponds to the direct decay of the $\omega$ meson. A mass resolution of $2 \%$ at the $\omega$ mass is extracted. The remaining signal continuum in this mass region may originate from in-medium $\rho$ decays.

To compare the spectral shapes of the $p+p$ and $p+A$ measured distributions, the $p+p$ data were rescaled to the $\mathrm{p}+\mathrm{Nb}$ results. The result is shown on the right panel of Fig. 5. Two scaling methods were used, showing consistent results. First is the ratio of $\pi^{0}$ produced in two systems (used in Fig. 5), estimated as $\sigma_{\pi^{0}}^{p N b} / \sigma_{\pi^{0}}^{p p}=31 \pm 6.5$. The second correction factor based on ratios of reaction cross sections and number of participants results in $\sigma_{\pi^{0}}^{p N b} / \sigma_{\pi^{0}}^{p p} \times\left\langle A>_{p N b}^{p a r t} /<A>_{p p}^{p a r t}=27.3\right.$. It is apparent from Fig. 5, that the $\mathrm{p}+\mathrm{Nb}$ and $\mathrm{p}+\mathrm{p}$ spectral shapes are almost identical without any mass dependency.

The situation changes considerably when one uses the HADES large momentum acceptance for $e^{+} e^{-}$pairs, and applies the condition on $e^{+} e^{-}$momenta. At high momenta $p_{e e}>0.8 \mathrm{GeV} / \mathrm{c}$ the spectral shapes are still very similar, while at lower momenta $p_{e e}<0.8 \mathrm{GeV} / \mathrm{c}$ the significant changes are apparent in region $M_{e e}>0.4 \mathrm{GeV} / \mathrm{c}^{2}$. The analysis is at its final stage, and the results 
will be published soon. Preliminarily, a strong excess yield is observed in $\mathrm{p}+\mathrm{Nb}$ system in the mass region where the $\rho$ meson is expected to be dominant. One can speculate about two possible reason for this effect. One is feeding of low momenta di-lepton pairs from the secondary interactions which produce baryonic resonances and $\rho$ meson, second is the $\rho$ spectral shape modification. A change of the $\omega$ line shape cannot be determined from the data due to very low yield in the $\omega$ mass peak in the $\mathrm{p}+\mathrm{Nb}$ system. The lower yield at the same time points to the $\omega$ collisonal broadening, and is in agreement with $\gamma$ induced reactions data [25].

\section{Summary}

Data on dielectron production from cold nuclear matter measured by the HADES spectrometer were presented. Comparing the $\mathrm{p}+\mathrm{Nb}$ data with the $\mathrm{p}+\mathrm{p}$ reference experiment results, a signifficant excess of low momenta dielectron pairs in the $\rho$ meson mass region is observed. At the same time, the $\omega$ yield seems to be lower in the pA system which is consistent with broadening of its spectral function in the medium.

We also presented data on the charged pion production. The results allowed for a reliable and precise normalization of the dielectron data, and contribute to the data from systematic studies of pion production in proton-nucleus collisions.

\section{Acknowledgement}

The collaboration gratefully acknowledges the support by LIP Coimbra (Portugal): PTDC/FIS/ 113339/2009, SIP JUC Cracow (Poland): N N202 286038 NN202198639, FZ Dresden-Rossendorf (FZD), Dresden (Germany): BMBF 06DR9059D, TU Munchen, Garching (Germany) MLLMunchen DFG EClust: 153VHNG-330, BMBF 06MT9156 TP5 TP6, GSI TMKrue 1012, NPI AS CR, Rez, Rez (Czech Republic): MSMT LC07050 GAASCR IAA100480803, USC - S. de Compostela, Santiago de Compostela (Spain): CPAN:CSD2007- 00042, Helmholtz alliance HA216/EMMI.

\section{References}

[1] S. Leupold, V. Metag, U. Mosel, Int. J. Mod. Phys. E19, 147-224 (2010)

[2] R. S. Hayano and T. Hatsuda, Rev. Mod. Phys. 82, 2949 (2010)

[3] B. Friman, C. Hohne, J. Knoll, S. Leupold, J. Randrup, R. Rapp and P. Senger, Lect. Notes Phys. 814, 11 (2011).

[4] M. Post, S. Leupold and U. Mosel, Nucl. Phys. A 741 81-148 (2004).

[5] G. Agakichiev et al., CERES Collaboration, Eur. Phys. J. C 41, 475, (2005).

[6] M. Masera, Nucl. Phys. A 590, 93c (1995).

[7] R. Arnaldi et al., NA60 collaboration, Phys. Rev. Lett. 96, 162302 (2006).

[8] R. J. Porter et al., DLS collaboration, Phys. Rev. Lett. 79, 1227 (1997).

[9] G. Agakishiev et al. (HADES Collaboration), Phys. Rev. Lett. 98, 052302 (2007).

[10] G. Agakishiev et al. (HADES Collaboration), Phys. Lett. B 663, 43 (2008). 
[11] G. Agakishiev et al. (HADES Collaboration), Phys. Rev. C, 014902 (2011).

[12] R. Nasseripour et al. (CLAS Collaboration), Phys. Rev. Lett. 99, 262302 (2007).

[13] M. Naruki et al., Phys. Rev. Lett. 96, 092301 (2006).

[14] M. Kotulla et al. (CBELSA-TAPS Collaboration), Phys. Rev. Lett. 100, 192302 (2008).

[15] T. Ishikawa et al. (SPring-8 Collaboration), Phys. Lett. B 608, 215-222 (2005).

[16] A. Polyanskiy et al. (ANKE Collaboration), Phys. Lett. B 695, 74 (2011).

[17] M. H. Wood et al. (CLAS Collaboration), Phys. Rev. Lett 105, 112301 (2010).

[18] M. Kaskulov, E. Hernandez and E. Oset, Eur. Phys. J. A 31, 245 (2007).

[19] G. Agakishiev et al. (HADES Collaboration) Eur. Phys. J. A41, 243 (2009).

[20] G. Agakishiev et al. (HADES Collaboration) arXiv:1112.3607 [nucl-ex], Eur. Phys. J. (2012) accepted for publication.

[21] A. Bolshakova et al. (HARP Collaboration), Eur. Phys. J. C62,697 (2009), Eur. Phys. J. C63, 549 (2009), Eur. Phys. J. C64,181 (2009), Eur. Phys. J. , C66, 57 (2009).

[22] M. Bleicher et al., J. Phys. G 25, 1859-189 (1999).

[23] Application Software Group, GEANT Detector Description and Simulation Tool, Program Library Long Writeups W5013, CERN, 3.21 edition, 1993.

[24] R. K. Tripathi, F. A. Cucinotta, J. W. Wilson, Nucl. Instr. and Meth. B117, 347 (1996).

[25] M. Nanova et al. (CBELSA-TAPS Collaboration), Phys. Rev. C 82, 035209 (2010). 